\title{
Network-Based Strategies Can Help Mono- and Poly-pharmacology Drug Discovery: A Systems Biology View
}

\author{
H. Billur Engin ${ }^{1}$, Attila Gursoy ${ }^{1}$, Ruth Nussinov ${ }^{2,3}$ and Ozlem Keskin ${ }^{1} *$
}

${ }^{1}$ Center for Computational Biology and Bioinformatics and College of Engineering, Koc University, Rumelifeneri Yolu, 34450 Sariyer Istanbul, Turkey; ${ }^{2}$ Center for Cancer Research Nanobiology Program, Frederick National Laboratory, National Cancer Institute, Frederick, MD 21702; ${ }^{3}$ Sackler Inst. Of Molecular Medicine, Sackler School of Medicine, Tel Aviv University, Tel Aviv 69978, Israel

\begin{abstract}
The cellular network and its environment govern cell and organism behavior and are fundamental to the comprehension of function, misfunction and drug discovery. Over the last few years, drugs were observed to often bind to more than one target; thus, polypharmacology approaches can be advantageous, complementing the "one drug - one target" strategy. Targeting drug discovery from the systems biology standpoint can help in studies of network effects of mono- and poly-pharmacology. In this mini-review, we provide an overview of the usefulness of network description and tools for mono- and poly-pharmacology, and the ways through which protein interactions can help single- and multi-target drug discovery efforts. We further describe how, when combined with experimental data, modeled structural networks which can predict which proteins interact and provide the structures of their interfaces, can model the cellular pathways, and suggest which specific pathways are likely to be affected. Such structural networks may facilitate structure-based drug design; forecast side effects of drugs; and suggest how the effects of drug binding can propagate in multi-molecular complexes and pathways.
\end{abstract}

Keywords: Poly-pharmacology, network pharmacology, protein-protein interaction inhibitors, systems biology, protein-protein interfaces, modeling.

\section{INTRODUCTION}

Early drug discovery sought "magic bullets"; i.e., maximally selective molecules for specific targets related to specific diseases. About 90 percent of the drugs designed following this paradigm fail in the late and most costly stages of clinical trials [1]. Developing a new drug takes up to 15-20 years [2], which highlights the need to broaden the current strategies. Drug discovery is challenging due to structural and sequence similarity among members of the same protein family which typically lead to active site conservation, and thus possible drug promiscuity; system diseases, which may relate to gene associations, redundancy in biological networks, and crosstalks between pathways, may make it even more complex. The cause of many common diseases (such as cancer, cardiovascular diseases and mental disorders) is not complications related to a single anomality, but rather is related to multiple molecular deviations [3]. Drugs designed with a "one-to-one" strategy may block the functions of their primary targets; however, at the same time they may lead to secondary reactions [4]. The increasing awareness of the high probability of such drug promiscuity has led to searches for new, enhanced strategies in drug discovery.

Target-centered drug discovery approaches are handicapped in foreseeing the outcome of drug regimen. The disturbance caused by a drug on an organism is not limited to only its targets; for instance, when human cancer cells were subject to drug treatment, the 1000 monitored (different) proteins in individual cells were all observed to be altered [5], illustrating the inherent difficulties in prediction of the outcome of drug-target interaction [6]. System-wide drug action may be better-understood using network analyses. There are approximately 25,000 genes in the human genome and 1,800 of them are known to be linked with various diseases, and this is likely to be an underestimation. The FDA approved drugs target around 400 gene products [7]. In the last decade, although the number of new

*Address correspondence to this author at the Center for Computational Biology and Bioinformatics and College of Engineering, Koc University, Rumelifeneri Yolu, 34450 Sariyer Istanbul, Turkey;

Tel:/Fax: ?????????????????; E-mail: okeskin @ku.edu.tr drugs increased, the number of drug targets did not rise as much. Marketed drugs generally interact with old targets [8]; thus, we would expect that more targets will be discovered as we delve into the human protein-protein interaction (PPI) network.

Below, we first provide an overview of poly-pharmacology followed by the cellular protein-protein interaction network, and its usefulness in drug discovery. We then describe how the modeling of the structural protein interaction networks, which predicts which proteins interact and how they interact, and uses this information together with experimental data to construct the cellular pathways can help drug discovery. Modeling the protein interactions and the structural pathways can help mono- and poly-pharmacology, an important area which currently attracts much interest.

\section{POLY-PHARMACOLOGY}

Poly-pharmacology searches for lead compounds that bind to multiple targets, and introduces a new concept of network pharmacology, which enlarges the 'drugome' [9]. It builds upon systems biology and drug discovery [10] aiming to treat diseases through multiple targets, which can be both a drug with several targets or a number of drugs with distinct targets. Poly-pharmacology describes and advocates consideration of a "many-to-many" relation between a ligand-protein couple, in contrast to a dominant "one drug-one target" drug design paradigm [11]. The novel computational approaches to poly-pharmacology have been reviewed by Xie et al. [12] recently.

\section{PPI TARGETING DRUGS}

Biological functions depend on signal transduction cascades [13]; and a large class of therapeutics target signaling [14]. Signaling typically takes place through PPIs. Blocking the information flow requires the design of (often) small molecules which inhibit these key interactions. A well-known example is heterotrimeric Gprotein subunit; designing inhibitors for G-protein-effectors or receptors can modulate GPCR signaling pathway.

Design of drugs that disrupt PPIs is known to be notoriously difficult. This is for two reasons: protein-protein interfaces have a 
more flat surface when compared to enzymes and usually do not have grooves which can serve as binding pockets [15]. The pockets on protein-protein interfaces are typically smaller than those in protein-ligand interactions [16] and difficult to drug. However, now it is becoming increasingly possible to overcome these handicaps, and PPI inhibitors are gaining importance as a class of drug targets [17]. One of the most important findings was the fact that interface regions usually contain clusters of residues, which are key contributors to the binding energy. These smaller regions of the interaction surface constitute "hot spots" [18-20]. Different studies showed that small molecules target hotspots on the protein-protein interfaces $[20,21]$. Hot spots on an interface may be predicted via HotPoint [22], HSPred [23], KFC [24], and additional servers. Moreover, currently allosteric drugs that bind elsewhere and lead to conformational changes in the interface appear increasingly feasible. Nonetheless, although designing drugs for disrupting protein-protein interactions has surged, some such drugs have long been in existence. Protease inhibitors are well-known marketed examples of this drug class [25]. The number of PPI targeting drugs is rising; examples include inhibitors targeting IL-2 [26], MDM2 [27, 28], BCL-2/BCL-XL[29], XIAP [30] and VLA-4 [30]. There are a number of reviews [13, 15, 25, 31-35], which investigate PPI inhibiting drugs, and these provide a more extensive list.

Pockets at the active sites of enzymes are typically stable, with high population times. Recently attempts to target PPI have also focused on detection and targeting of transient dynamic pockets, which may be stabilized upon drug binding. Such grooves can be found in PPIs [36] and elsewhere on protein surfaces and can serve as orthosteric and allosteric binding sites. Transient pockets occur often [37]; the question is their size and population time. Furthermore, the surface of interacting proteins is flexible and some disordered proteins can only be solved upon interaction with their partners [38]. Their flexibility implicates formation of transient pockets, which are very useful for inhibitor design [39]. A number of drugs have been reported to stick to these transient pockets on the surface of protein interfaces [40]. Metz et al. [41] proposed a tool that locates transient pockets of PPIs on the basis of geometry, and molecular dynamics simulation protocols are also being developed toward this aim. Further validation of the presence of at least small pockets comes from a computational analysis of crystal structures. This study observed that among 18 protein-protein complexes, 16 contain pre-existing pockets in their unbound structures [42].

A number of clinical therapies are based on humanized monoclonal antibodies which disrupt PPI. These therapies have high specificity and low toxicity; however, they also have some deficiencies such as lack of cell/blood-brain barrier permeability, and poor oral bioavailability. Thus, humanized monoclonal antibodies therapies may not be broadly applicable to PPI inhibitor design [13] in the near future.

The data on PPI inhibitors has been compiled in databases and literature. One of these, 2P2I [43], is a database which provides structural data for a collection of protein-protein interfaces with known inhibitors. TIMBAL [44] is also a database where one can find small molecules disrupting PPIs. Furthermore, Sali et. al. spotted "bi-functional positions" of proteins (overlapping ligand and protein binding sites) by aligning homologous proteins. They pointed out to the significant number of proteins that have such bifunctional positions and released the collection of structurally characterized modulators of protein interactions at http: //pibase.janelia.org [45].

\section{DRUGS TARGETING MULTIPLE PROTEINS}

Side effects are one of the main reasons for drug failure [46]. In the last 10 years nearly 20 drugs have been banned from the market for causing severe side effects [47]. Adverse effects can be caused by the inherent mechanism of action of a drug, by toxic metabolites following drug degradation and by unpredictable side effects due to "off-targets" of drug interactions. A number of studies highlight the promiscuity as a common attribute of drugs. Yildirim et al. [8] constructed a drug-target network from 4252 drugs targeting $394 \mathrm{hu}$ man proteins. They found out that among 890 drugs 788 had at least one common target and in that network the average number of target proteins was 1.8 per drug. This result revealed the fact that new drugs generally target known druggable proteins and that the number of drugs targeting others is low in the market. However a more recent study by Mestres et al. [48], updated the average number of target proteins per drug as 6.3 , which points to the high tendency of drugs to be multi-targeted. Paolini et al. [49] searched for the extent of promiscuity in the global pharmacological space and they also observed that among 276,122 active drug compounds, $35 \%$ hit multiple targets. The data compiled in the drug-target databases also indicates this many-to-many behavior. Some of these databases are listed in the Table 1. Among the multi-target drug examples, there are a number of kinase inhibitors, which operate by affecting multiple targets $[50,51]$ and the anticancer drug lenalidomide [12]. Several multi-target drugs were also discovered by chance [52].

There are examples suggesting that targeting multiple proteins simultaneously may be successful, such as non-steroidal antiinflammatory drugs (NSAIDs); antidepressants; multi-target kinase inhibitors and anticancer drugs [53]. Drug combinations ("cocktails') may bind at different sites on the same protein; or to multiple different proteins. Examples include the three drugs combination used to treat HIV infection, which is composed of reversetranscriptase and protease inhibitors [6] and the drug combinations known as "CHOP", which is used in the treatment of nonHodgkin's lymphoma [54]. Another synergistic drug combination example is Cytarabine and Aplidin (Fig. 1), used for enhancing their antitumor activities in leukaemia and lymphoma models [55]. Cytarabine is an anticancer drug used in the treatment of patients with leukemia [56] and Aplidin is another, which activates EGFR, Src, JNK and p38MAPK [57] and inhibits VEGF [58]. When system-wide effect of Aplidin is investigated, it is observed to activate death receptor of Fas ligands [59]. This outcome of Aplidin may be due to the activation of JNK/p38 MAPK pathway [60]. In turn, Fas ligand activates the receptor-mediated extrinsic cascade of apoptosis [61]. In addition, Cytarabine increases cellular stress by inhibiting DNA repair and RNA synthesis and drops the MCL1 level which leads to activation of CASPs [62]. Finally CASPs triggers the apoptosis viathe mitochondrial intrinsic cascade [61].

Due to drug combinations' condensed side effects and enhanced treatment capability, understanding the underlying mechanisms is an important and interesting task, as well as detecting new applicable drug cocktails. There is an increasing number of publications in this area; such as the "drug cocktail network" built in order to investigate existing drug cocktails and identifying new ones, in a recent work [63]. They mentioned that drugs in a cocktail tend to interact with same partners and share common therapeutic effects. Another example is the computational method Zhao et al. [64] used for inferring new drug combinations. They combined molecular and pharmacological properties of drugs for this purpose and looked for feature patterns enriched in drug combinations. Predictions of their method were $69 \%$ supported by literature. Moreover, they have also found important clues about the working principles of combinatorial therapies. "Combinatorial Drug Assessment"[65] is an alternative tool for combinatorial drug discovery, which uses gene expression profiling and multiple signaling pathways. Lastly Wang et al. [66] considered drug combinations in the perspective of genetic interaction network and associated human pathways. They found that drug combinations alter functionally correlated pathways and have a smaller influence range in the genetic interaction networks.

\section{A SYSTEMS BIOLOGY VIEW}

Biological systems are governed by physical and functional interactions. Systems biology simulates and orchestrates the mole- 
Table 1. List of some drug-target databases.

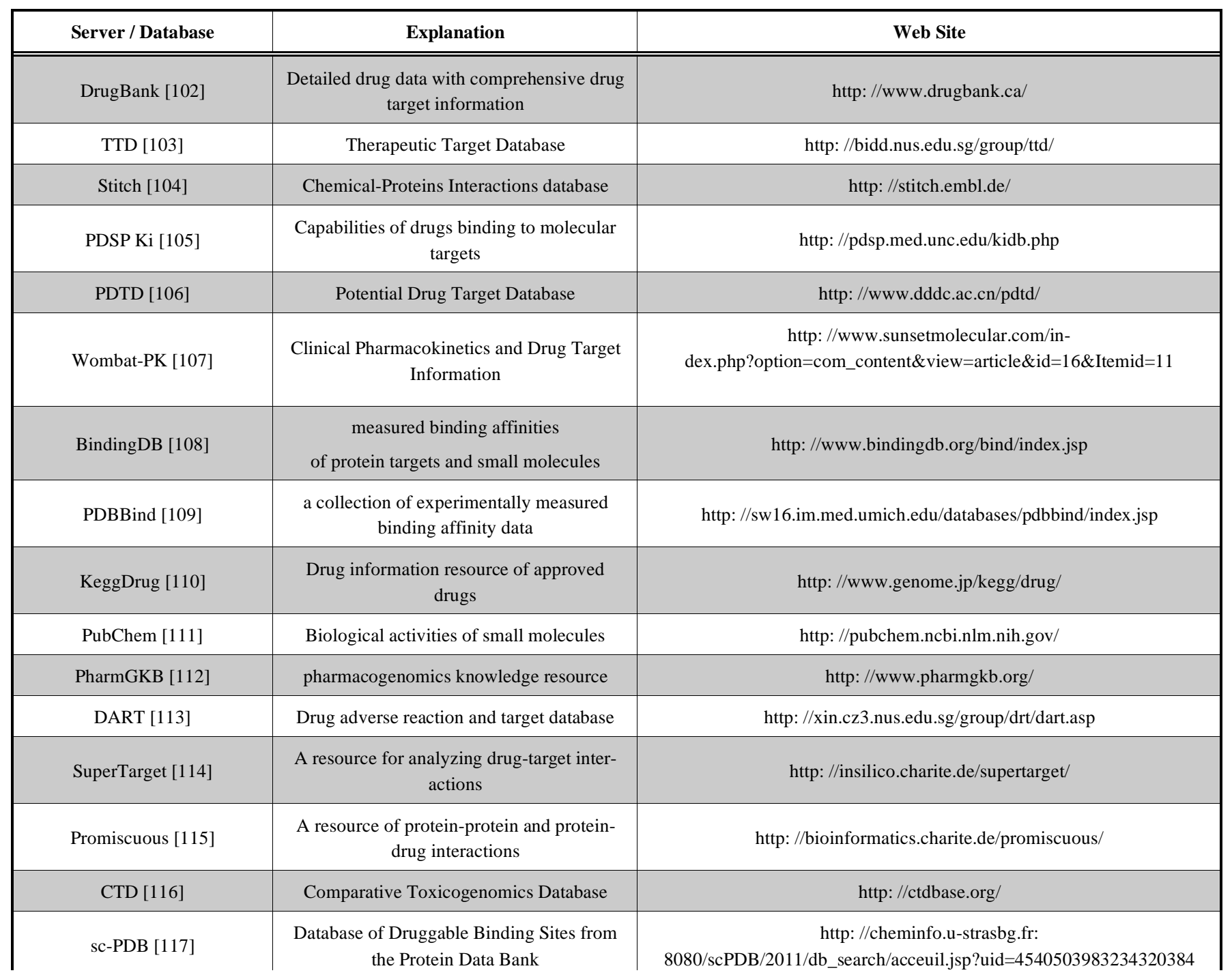

cules to optimally adapt the organism response to its environment. Diseases disturb the network; 'good' drugs restore the network to its 'proper' desired state [67]. Network descriptions and analyses are important tools in systems biology; they are powerful in abstracting the complex relationships inside cells and between them, and they often provide clues for drug discovery [68]. While data are incomplete, and the approaches may not have matured, network descriptions and tools are gradually becoming common place [69].

The human protein-protein interaction (PPI) network is huge with approximately 130,000 binary interactions between proteins [70], and is expected to be far larger, with around 650,000 PPIs [71]. Protein-protein interaction networks are of vast importance in medicine $[72,73]$. From the drug development standpoint, we would expect it to have critical components, which would make enticing drug targets. Network topology may help, because drug targets are usually not arbitrarily located on the protein interaction networks [74]. Drugs that perturb topologically critical nodes (such as highly connected nodes) have increased risk of causing lethality [75], while blocking the targeted function. That's why marketed drugs do not generally target high degree nodes [76]. An 'ideal' drug target would have fewer neighbors while being located at some strategic point of the human disease network [6]. Such a target may be a non-vital bridging node [77]. It may disturb the information flow and the disease process while not causing serious side effects. For complex diseases like cardiovascular disease, central nervous system disorders, cancer, Alzheimer and aging, a network perspective is critically important. These require consideration of the global map of protein interactions and estimation of the expected outcome on the multiple, inter-connected pathways. As we describe below, in some diseases that are resistant to drug therapy [78], network-based strategy, where another protein in the same pathway is targeted may suggest alternative targets that may lead to the sought outcome $[79,80]$.

When enriched by high-throughput data, networks may obtain possible responses to a drug or optimum combination of drugs for reaching a desired outcome. On the other hand, because such data is derived from population of cells, its accuracy for specific environments and physiological states may be compromised. Networks may be analyzed using mathematical models such as Flux Balance Analysis [43, 81-83], differential equations [84], Petri Nets [85], Integer Linear Programming [86] and Boolean logic gates [87].

Another use of networks is its topological properties such as hubs, betweenness, modules, etc.. Network topology determines the information flow. Information flow and robustness analyses are used to locate essential components. These algorithms are utilized to find perturbed proteins by hypothetical drugs [88] or for locating optimum drug targets that have little influence on other functions, 


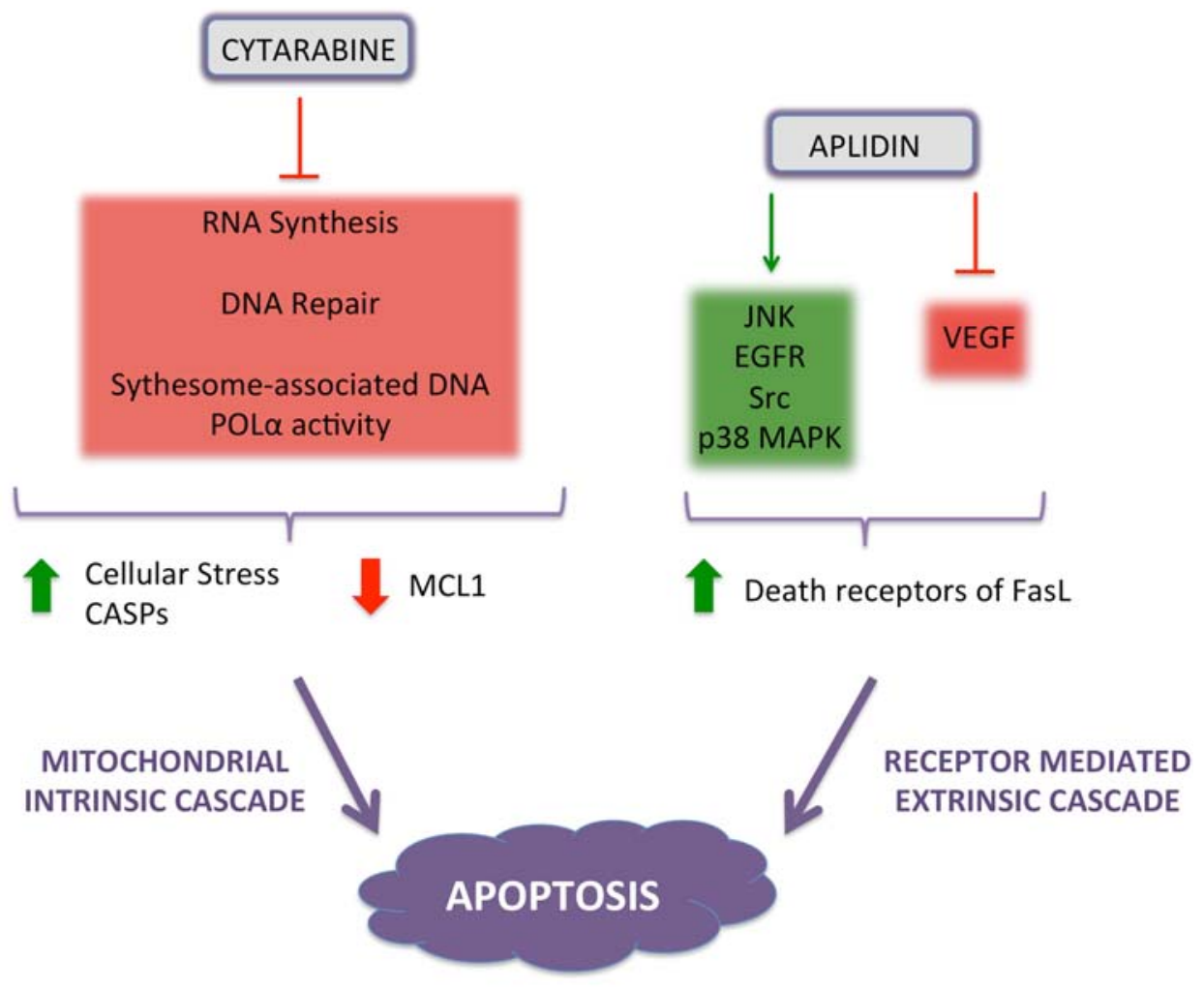

Fig. (1). Synergistic drug combination of Cytarabine and Aplidinenhances antitumor activities.

apart from the intended one [89-92]. A key question is how to choose an efficient combination of multiple drug targets; especially those that while not key players in central pathways, ensure information flow among the network elements [77].

\section{THE ADVANTAGES AND HANDICAPS OF MODELED PROTEIN-PROTEIN INTERACTIONS IN MONO- AND POLY-PHARMACOLOGY}

Key requirements in drug discovery are the availability of protein structures and their interactions; the pathways in which they are located and the pathway cross-talk; and how similar are the binding sites to which they bind to those of other proteins in the cell. Modeling protein interactions can help by predicting which proteins interact, which permits the construction of more complete pathways and the cellular network. These may allow prediction of how targeting a specific protein can affect the entire system. The PRISM server $[93,94]$ is one of the tools which predicts the interacting protein couples and their interface structures. Further, because the modeled interactions provide also the information on how the proteins interact, they allow prediction of which partners interact through the same binding site. Thus, if a particular protein is targeted, this may abolish the competitive binding at the same shared site, driving the system in a certain direction, which the structural network may forecast. Such predictions may be particularly powerful for multi-molecular complexes, which are prone to toxic side effects. If the drugs target certain PPIs, the structural network may suggest the other PPI which share similar motifs [95]; and as such, may also be affected by the drug, which may also lead to toxic side effects.

Networks may be used to explain side effects of multi-target drugs. Xie et al. [96] studied the side effects of torcetrapib, which is an inhibitor of cholesteryl ester transfer protein (CETP). Torcetrapib was a proposed treatment for cardiovascular disease and was in clinical trials. The authors compared all ligand-binding sites in all available protein structures, with the pockets on torcetrapib and created an off-target binding network. They combined their study with biological pathways and found the likely reasons for the effects of torcetrapib on blood pressure.

Propagation of the effects of drugs in the network may be observed for orthosteric and allosteric drugs [97, 98]. In the case of orthosteric drugs, which block the protein active site, the protein is impaired and its function is abolished; in the case of allosteric drugs, the modulating effects of drugs propagate through the protein and, through the protein-protein interactions, across the pathways. However, the effects are likely to be strongest in proteins sharing the same complex.

A key handicap of modeled structural networks is that they provide a static view of cell, and of the proteins. Yet, the cellular network is highly dynamic; proteins associate and dissociate. This is challenging to model, because the affinities of their interactions which are typically measured in solution, do not necessarily reflect the in vivo environment, where the affinities at one binding site are affected by prior allosteric events at different sites, for example, binding of other partners or post-translational modifications. They also may not account for the presence of co-factors; and fluctuations in the environment. An additional challenging problem is protein dynamics; protein structures fluctuate, and the distributions of their conformational ensembles change dynamically, which affects the binding site conformations and drug binding [97]. Accounting for dynamics in the proteins and across the pathways and the network is an extremely challenging problem. This is because it both necessitates detailed experimental data and highly demanding computational requirements. To date, modeling on the network scale is not able to fully address these problems [68]. However, for specific proteins, on the local scale, Nuclear Magnetic Resonance (NMR) and molecular dynamic simulations may be able to provide some clues.

Despite these shortcomings, single- and multi-drug pharmacology can benefit from the modeled structural proteome. Predictions 
are able to provide leads and hypotheses, which can then be validated by experiment.

\section{CONCLUSIONS}

The network-like structure established by the interactions of proteins has been discovered already some years ago [99, 100]; and although there have been many PPI curation studies and considerable research, the human PPI network is still believed to be lacking important interactions [70]. The success of network-based methods is tightly connected to the completeness of data; and as pointed out in a recent review [101] databases are far from being complete. As more protein interactions are discovered, systems biology will be able to simulate the mechanism inside and between the cells more effectively. Structural data of single proteins and their complexes is also expected to increase. As a consequence, complete models of systems biology and structural pathways will hopefully pave a path to tailored patient-specific treatments.

\section{CONFLICT OF INTEREST}

The authors confirm that this article content has no conflicts of interest.

\section{ACKNOWLEDGEMENTS}

This work has been supported by TUBITAK (Research Grant Numbers: 109T343 and 109E207). This project has been funded in whole or in part with Federal funds from the National Cancer Institute, National Institutes of Health, under contract number HHSN261200800001E. The content of this publication does not necessarily reflect the views or policies of the Department of Health and Human Services, nor does mention of trade names, commercial products, or organizations imply endorsement by the U.S. Government. This research was supported (in part) by the Intramural Research Program of the NIH, National Cancer Institute, Center for Cancer Research.

\section{REFERENCES}

[1] Nwaka S, Hudson A. Innovative lead discovery strategies for tropical diseases. Nat Rev Drug Discov 2006; 5: 941-55.

[2] O'Connor KA, Roth BL. Finding new tricks for old drugs: an efficient route for public-sector drug discovery. Nat Rev Drug Discov 2005; 4: 1005-14.

[3] Pujol A, Mosca R, Farres J, Aloy P. Unveiling the role of network and systems biology in drug discovery. Trends Pharmacol Sci 2010; 31: 115-23.

[4] Giacomini KM, Krauss RM, Roden DM, Eichelbaum M, Hayden MR, Nakamura Y. When good drugs go bad. Nature 2007; 446: 975-7.

[5] Cohen AA, Geva-Zatorsky N, Eden E, et al. Dynamic proteomics of individual cancer cells in response to a drug. Science 2008; 322: 1511-6.

[6] Fliri AF, Loging WT, Volkmann RA. Cause-effect relationships in medicine: a protein network perspective. Trends Pharmacol Sci 2010; 31: 547-55.

[7] Wist AD, Berger SI, Iyengar R. Systems pharmacology and genome medicine: a future perspective. Genome Med 2009; 1: 11.

[8] Yildirim MA, Goh KI, Cusick ME, Barabasi AL, Vidal M. Drugtarget network. Nat Biotechnol 2007; 25: 1119-26.

[9] Hopkins AL. Network pharmacology: the next paradigm in drug discovery. Nat Chem Biol 2008; 4: 682-90.

[10] Boran AD, Iyengar R. Systems approaches to polypharmacology and drug discovery. Current Opinion Drug Discov Develop 2010; 13: 297-309.

[11] Brown JB, Okuno Y. Systems biology and systems chemistry: new directions for drug discovery. Chem Biol 2012; 19: 23-8.

[12] Xie L, Kinnings SL, Bourne PE. Novel computational approaches to polypharmacology as a means to define responses to individual drugs. Annual Rev Pharmacol Toxicol 2012; 52: 361-79.

[13] Blazer LL, Neubig RR. Small molecule protein-protein interaction inhibitors as CNS therapeutic agents: current progress and future hurdles. Neuropsychopharmacology : official Pub Am College Neuropsychopharmacol 2009; 34: 126-41.
[14] Brunton LL LJ, Parker KL. Goodman \& Gilman's: The Pharmacological Basis of Therapeutics. New York: McGraw-Hill Companies, Inc 2006.

[15] Arkin MR, Wells JA. Small-molecule inhibitors of protein-protein interactions: progressing towards the dream. Nat Rev Drug Discov 2004; 3: 301-17.

[16] Fuller JC, Burgoyne NJ, Jackson RM. Predicting druggable binding sites at the protein-protein interface. Drug Discov Today 2009; 14 : 155-61.

[17] Wanner J, Fry DC, Peng Z, Roberts J. Druggability assessment of protein-protein interfaces. Future medicinal chemistry 2011; 3: 2021-38.

[18] Clackson T, Wells JA. A hot spot of binding energy in a hormonereceptor interface. Science 1995, 267: 383-6.

[19] Acuner Ozbabacan SE, Gursoy A, Keskin O, Nussinov R. Conformational ensembles, signal transduction and residue hot spots: application to drug discovery. Current Opinion Drug Discov Develop 2010; 13: 527-37.

[20] Wells JA, McClendon CL. Reaching for high-hanging fruit in drug discovery at protein-protein interfaces. Nature 2007; 450: 1001-9.

[21] Thangudu RR, Bryant SH, Panchenko AR, Madej T. Modulating protein-protein interactions with small molecules: the importance of binding hotspots. J Mol Biol 2012; 415: 443-53.

[22] Tuncbag N, Keskin O, Gursoy A. HotPoint: hot spot prediction server for protein interfaces. Nucleic Acids Res 2010; 38: W402-6.

[23] Lise S, Archambeau C, Pontil M, Jones DT. Prediction of hot spot residues at protein-protein interfaces by combining machine learning and energy-based methods. BMC Bioinformatics 2009; 10: 365 .

[24] Darnell SJ, LeGault L, Mitchell JC. KFC Server: interactive forecasting of protein interaction hot spots. Nucleic Acids Res 2008; 36: W265-9.

[25] Vselovsky AVA, A. I. . Inhibitors of Protein-Protein Interactions as Potential Drugs. Curr Computer-Aided Drug Des 2007: 51-8.

[26] Braisted AC, Oslob JD, Delano WL, et al. Discovery of a potent small molecule IL-2 inhibitor through fragment assembly. J Am Chem Soc 2003; 125: 3714-5.

[27] Vu BT, Vassilev L. Small-molecule inhibitors of the p53-MDM2 interaction. Curr Topics Microbiol Immunol 2011; 348: 151-72.

[28] Canner JA, Sobo M, Ball S, et al. MI-63: a novel small-molecule inhibitor targets MDM2 and induces apoptosis in embryonal and alveolar rhabdomyosarcoma cells with wild-type p53. Br J Cancer 2009; 101: 774-81.

[29] Tse C, Shoemaker AR, Adickes J, et al. ABT-263: a potent and orally bioavailable Bcl-2 family inhibitor. Cancer Res 2008; 68: 3421-8.

[30] Straub CS. Targeting IAPs as an approach to anti-cancer therapy. Curr Topics Med Chem 2011; 11: 291-316.

[31] Silviya D. Furdas LC, Wolfgang Sippl and Manfred Jung. Inhibition of bromodomain-mediated protein-protein interactions as a novel therapeutic strategy. Med Chem Commun 2012: 123-34.

[32] Busschots K, De Rijck J, Christ F, Debyser Z. In search of small molecules blocking interactions between HIV proteins and intracellular cofactors. Mol Biosystems 2009; 5: 21-31.

[33] Buchwald P. Small-molecule protein-protein interaction inhibitors: therapeutic potential in light of molecular size, chemical space, and ligand binding efficiency considerations. IUBMB life 2010;62: 724-31.

[34] Sperandio O, Reynes CH, Camproux AC, Villoutreix BO. Rationalizing the chemical space of protein-protein interaction inhibitors. Drug Discov Today 2010; 15: 220-9.

[35] Arkin MR, Whitty A. The road less traveled: modulating signal transduction enzymes by inhibiting their protein-protein interactions. Curr Opinion Chemical Biol 2009; 13: 284-90.

[36] Keskin O, Gursoy A, Ma B, Nussinov R. Principles of proteinprotein interactions: what are the preferred ways for proteins to interact? Chemical Rev 2008; 108: 1225-44.

[37] Arkin MR, Randal M, DeLano WL, et al. Binding of small molecules to an adaptive protein-protein interface. Proc Nat Acad Sci USA 2003; 100: 1603-8.

[38] Gao M, Skolnick J. Structural space of protein-protein interfaces is degenerate, close to complete, and highly connected. Proc Natl Acad Sci USA 2010; 107: 22517-22.

[39] Eyrisch S, Helms V. Transient pockets on protein surfaces involved in protein-protein interaction. J Med Chem 2007; 50: 3457-64. 
[40] Eyrisch S, Helms V. What induces pocket openings on protein surface patches involved in protein-protein interactions? J Computer-Aided Molecular Des 2009; 23: 73-86.

[41] Metz A, Pfleger C, Kopitz H, Pfeiffer-Marek S, Baringhaus KH, Gohlke H. Hot spots and transient pockets: predicting the determinants of small-molecule binding to a protein-protein interface. J Chemical Information Mod 2012; 52: 120-33.

[42] Li X, Keskin O, Ma B, Nussinov R, Liang J. Protein-protein interactions: hot spots and structurally conserved residues often locate in complemented pockets that pre-organized in the unbound states: implications for docking. J Mol Biol 2004; 344: 781-95.

[43] Bourgeas R, Basse MJ, Morelli X, Roche P. Atomic analysis of protein-protein interfaces with known inhibitors: the 2P2I database. PloS one 2010; 5: e9598.

[44] Higueruelo AP, Schreyer A, Bickerton GR, Pitt WR, Groom CR, Blundell TL. Atomic interactions and profile of small molecules disrupting protein-protein interfaces: the TIMBAL database. Chemical Biol Drug Des 2009; 74: 457-67.

[45] Davis FP, Sali A. The overlap of small molecule and protein binding sites within families of protein structures. PLoS Computational Biol 2010; 6: e1000668.

[46] Scheiber J, Chen B, Milik M, et al. Gaining Insight into Off-Target Mediated Effects of Drug Candidates with a Comprehensive Systems Chemical Biology Analysis. J Chem Inf Model 2009.

[47] Scheiber J, Jenkins JL, Sukuru SC, et al. Mapping adverse drug reactions in chemical space. J Med Chem 2009; 52: 3103-7.

[48] Mestres J, Gregori-Puigjane E, Valverde S, Sole RV. Data completeness--the Achilles heel of drug-target networks. Nature Biotechnol 2008; 26: 983-4.

[49] Paolini GV, Shapland RH, van Hoorn WP, Mason JS, Hopkins AL. Global mapping of pharmacological space. Nature Biotechnol 2006; 24: 805-15.

[50] Hopkins AL, Mason JS, Overington JP. Can we rationally design promiscuous drugs? Curr Opinion Structural Biol 2006; 16: 12736 .

[51] Hopkins AL. Network pharmacology. Nat Biotechnol 2007; 25: 1110-1.

[52] Lange RP, Locher HH, Wyss PC, Then RL. The targets of currently used antibacterial agents: lessons for drug discovery. Curr Pharm Des 2007; 13: 3140-54.

[53] Knight ZA, Lin H, Shokat KM. Targeting the cancer kinome through polypharmacology. Nat Rev Cancer 2010; 10: 130-7.

[54] Dixon SJ, Stockwell BR. Drug discovery: engineering drug combinations. Nat chemical Biol 2010; 6: 318-9.

[55] Humeniuk R, Menon LG, Mishra PJ, et al. Aplidin synergizes with cytosine arabinoside: functional relevance of mitochondria in Aplidin-induced cytotoxicity. Leukemia : official $\mathrm{J}$ the Leukemia Society of America, Leukemia Research Fund, UK 2007; 21: 2399405 .

[56] Pelicano H, Carew JS, McQueen TJ, et al Targeting Hsp90 by 17AAG in leukemia cells: mechanisms for synergistic and antagonistic drug combinations with arsenic trioxide and Ara-C. Leukemia : official $\mathrm{J}$ the Leukemia Society of America, Leukemia Research Fund, UK 2006; 20: 610-9.

[57] Cuadrado A, Gonzalez L, Suarez Y, Martinez T, Munoz A. JNK activation is critical for Aplidin-induced apoptosis. Oncogene 2004; 23: 4673-80.

[58] Biscardi M, Caporale R, Balestri F, Gavazzi S, Jimeno J, Grossi A. VEGF inhibition and cytotoxic effect of aplidin in leukemia cell lines and cells from acute myeloid leukemia. Annals of oncology : official J Eur Soc Med Oncol/ ESMO 2005; 16: 1667-74.

[59] Gajate C, Mollinedo F. Cytoskeleton-mediated death receptor and ligand concentration in lipid rafts forms apoptosis-promoting clusters in cancer chemotherapy. J Biological Chem 2005; 280: 11641-7.

[60] Mansouri A, Ridgway LD, Korapati AL, et al. Sustained activation of JNK/p38 MAPK pathways in response to cisplatin leads to Fas ligand induction and cell death in ovarian carcinoma cells. J Biological Chem 2003; 278: 19245-56.

[61] Jia J, Zhu F, Ma X, Cao Z, Li Y, Chen YZ. Mechanisms of drug combinations: interaction and network perspectives. Nat Rev Drug Discov 2009; 8: 111-28

[62] de Vries JF, Falkenburg JH, Willemze R, Barge RM. The mechanisms of Ara-C-induced apoptosis of resting B-chronic lymphocytic leukemia cells. Haematologica 2006; 91: 912-9.
[63] Xu KJ, Song J, Zhao XM. The drug cocktail network. BMC Systems Biol 2012; 6 Suppl 1: S5.

[64] Zhao XM, Iskar M, Zeller G, Kuhn M, van Noort V, Bork P. Prediction of drug combinations by integrating molecular and pharmacological data. PLoS Computational Biol 2011; 7: e1002323.

[65] Lee JH, Kim DG, Bae TJ, et al. CDA: combinatorial drug discovery using transcriptional response modules. PloS one 2012; 7: e42573.

[66] Wang YY, Xu KJ, Song J, Zhao XM. Exploring drug combinations in genetic interaction network. BMC Bioinformatics 2012; 13 Suppl 7: S7.

[67] Azmi AS, Wang Z, Philip PA, Mohammad RM, Sarkar FH. Proof of concept: network and systems biology approaches aid in the discovery of potent anticancer drug combinations. Mol Cancer Therapeut 2010; 9: 3137-44.

[68] Keskin O, Gursoy A, Ma B, Nussinov R. Towards drugs targeting multiple proteins in a systems biology approach. Curr Topics Med Chem 2007; 7: 943-51.

[69] Faratian D, Clyde RG, Crawford JW, Harrison DJ. Systems pathology--taking molecular pathology into a new dimension. Nat Rev Clinical Oncol 2009; 6: 455-64.

[70] Venkatesan K, Rual JF, Vazquez A, et al. An empirical framework for binary interactome mapping. Nature Method 2009; 6: 83-90.

[71] Stumpf MP, Thorne T, de Silva E, et al. Estimating the size of the human interactome. Proc Natl Acad Sci USA 2008; 105: 6959-64.

[72] Ruffner H, Bauer A, Bouwmeester T. Human protein-protein interaction networks and the value for drug discovery. Drug Discov Today 2007; 12: 709-16

[73] Ideker T, Sharan R. Protein networks in disease. Genome Res 2008; 18: 644-52.

[74] Ma'ayan A, Jenkins SL, Goldfarb J, Iyengar R. Network analysis of FDA approved drugs and their targets. The Mount Sinai J medicine, New York 2007; 74: 27-32.

[75] Jeong H, Mason SP, Barabasi AL, Oltvai ZN. Lethality and centrality in protein networks. Nature 2001; 411: 41-2.

[76] Hase T, Tanaka H, Suzuki Y, Nakagawa S, Kitano H. Structure of protein interaction networks and their implications on drug design. PLoS Computational Biol 2009; 5: e1000550.

[77] Hwang WC, Zhang A, Ramanathan M. Identification of information flow-modulating drug targets: a novel bridging paradigm for drug discovery. Clin Pharmacol Therapeut 2008; 84: 563-72.

[78] Kitano H. Cancer Robustness and Therapy Strategies. In cancer systems biology, bioinformatics and medicine. Springer; 2011

[79] Stein A, Pache RA, Bernado P, Pons M, Aloy P. Dynamic interactions of proteins in complex networks: a more structured view. FEBS J 2009; 276: 5390-405.

[80] Pache RA, Zanzoni A, Naval J, Mas JM, Aloy P. Towards a molecular characterisation of pathological pathways. FEBS letters 2008; 582: 1259-65.

[81] Kinnings SL, Xie L, Fung KH, Jackson RM, Bourne PE. The Mycobacterium tuberculosis drugome and its polypharmacological implications. PLoS Comput Biol 2010; 6: e1000976.

[82] Dasika MS, Burgard A, Maranas CD. A computational framework for the topological analysis and targeted disruption of signal transduction networks. Biophys J 2006; 91: 382-98.

[83] Lee JM, Gianchandani EP, Papin JA. Flux balance analysis in the era of metabolomics. Brief Bioinform 2006; 7: 140-50.

[84] McAdams HH, Shapiro L. Circuit simulation of genetic networks. Science 1995, 269: 650-6.

[85] Peleg M, Rubin D, Altman RB. Using Petri Net tools to study properties and dynamics of biological systems. J Am Med Inform Assoc 2005; 12: 181-99.

[86] Li Z, Wang RS, Zhang XS, Chen L. Detecting drug targets with minimum side effects in metabolic networks. IET Syst Biol 2009; 3: 523-33.

[87] Layek R, Datta A, Bittner M, Dougherty ER. Cancer therapy design based on pathway logic. Bioinformatics 2011; 27: 548-55.

[88] Ruths D, Muller M, Tseng JT, Nakhleh L, Ram PT. The signaling petri net-based simulator: a non-parametric strategy for characterizing the dynamics of cell-specific signaling networks. PLoS computational Biol 2008; 4: e1000005.

[89] Dasika MS, Burgard A, Maranas CD. A computational framework for the topological analysis and targeted disruption of signal transduction networks. Biophys J 2006; 91: 382-98. 
[90] Sridhar P, Kahveci T, Ranka S. An iterative algorithm for metabolic network-based drug target identification. Pacific Symposium on Biocomputing Pacific Symposium Biocomputing 2007: 88-99.

[91] Sridhar P, Song B, Kahveci T, Ranka S. Mining metabolic networks for optimal drug targets. Pacific Symposium on Biocomputing Pacific Symposium Biocomput 2008: 291-302.

[92] Yang K, Bai H, Ouyang Q, Lai L, Tang C. Finding multiple target optimal intervention in disease-related molecular network. Mol Systems Biol 2008; 4: 228.

[93] Tuncbag N, Gursoy A, Nussinov R, Keskin O. Predicting proteinprotein interactions on a proteome scale by matching evolutionary and structural similarities at interfaces using PRISM. Nat Protocols 2011; 6: 1341-54.

[94] Ogmen U, Keskin O, Aytuna AS, Nussinov R, Gursoy A. PRISM: protein interactions by structural matching. Nucleic Acids Res 2005; 33: W331-6.

[95] Engin HB, Keskin O, Nussinov R, Gursoy A. A strategy based on protein-protein interface motifs may help in identifying drug offtargets. J Chem Inf Mod 2012; 52: 2273-86.

[96] Xie L, Li J, Bourne PE. Drug discovery using chemical systems biology: identification of the protein-ligand binding network to explain the side effects of CETP inhibitors. PLoS Comput Biol 2009; 5: e1000387.

[97] Kar G, Keskin O, Gursoy A, Nussinov R. Allostery and population shift in drug discovery. Curr Opinion Pharmacol 2010; 10: 715-22.

[98] Nussinov R, Tsai CJ, Csermely P. Allo-network drugs: harnessing allostery in cellular networks. Trends Pharmacological Sci 2011; 32: 686-93.

[99] Schachter V. Protein-interaction networks: from experiments to analysis. Drug Discov Today 2002; 7: S48-54.

[100] Schwikowski B, Uetz P, Fields S. A network of protein-protein interactions in yeast. Nat Biotechnol 2000; 18: 1257-61.

[101] Blow N. Systems biology: Untangling the protein web. Nature 2009; 460: 415-8.

[102] Knox C, Law V, Jewison T, et al. DrugBank 3.0: a comprehensive resource for 'omics' research on drugs. Nucleic Acids Res 2011; 39: D1035-41.

[103] Zhu F, Shi Z, Qin C, et al. Therapeutic target database update 2012: a resource for facilitating target-oriented drug discovery. Nucleic Acids Res 2012; 40: D1128-36.

[104] Kuhn M, Szklarczyk D, Franceschini A, et al. STITCH 2: an interaction network database for small molecules and proteins. Nucleic Acids Res 2010; 38: D552-6.
[105] BL Roth WK, Patel S, E Lopez. The Multiplicity of Serotonin Receptors: Uselessly diverse molecules or an embarrasment of riches?. Neuroscientist 2000; 6: 252-62.

[106] Gao Z, Li H, Zhang H, et al. PDTD: a web-accessible protein database for drug target identification. BMC Bioinformatics 2008; 9: 104

[107] Oprea TI, Berellini G, Olah M, Fejgin K, Boyer S. Rapid ADME Filters for Lead Discovery. In Molecular Interaction Fields. New York: Wiley-VCH; 2006 249-72

[108] Liu T, Lin Y, Wen X, Jorissen RN, Gilson MK. BindingDB: a web-accessible database of experimentally determined proteinligand binding affinities. Nucleic Acids Res 2007; 35: D198-201.

[109] Wang R, Fang X, Lu Y, Yang CY, Wang S. The PDBbind database: methodologies and updates. J Med Chem 2005; 48: 41119.

[110] Kanehisa M, Goto S, Hattori M, et al. From genomics to chemical genomics: new developments in KEGG. Nucleic Acids Res 2006; 34: D354-7.

[111] Bolton E WY, Thiessen PA, Bryant SH. PubChem: Integrated Platform of Small Molecules and Biological Activities. In Annua Reports in Computational Chemistry. Volume 4. Washington, DC: American Chemical Society; 2008

[112] McDonagh EM, Whirl-Carrillo M, Garten Y, Altman RB, Klein TE. From pharmacogenomic knowledge acquisition to clinical applications: the PharmGKB as a clinical pharmacogenomic biomarker resource. Biomarkers Med 2011; 5: 795-806.

[113] Ji ZL, Han LY, Yap CW, Sun LZ, Chen X, Chen YZ. Drug Adverse Reaction Target Database (DART) : proteins related to adverse drug reactions. Drug safety : an Int J Med Toxicol Drug Experience 2003; 26: 685-90.

[114] Hecker N, Ahmed J, von Eichborn J, et al. SuperTarget goes quantitative: update on drug-target interactions. Nucleic acids research 2012; 40: D1113-7.

[115] von Eichborn J, Murgueitio MS, Dunkel M, Koerner S, Bourne PE Preissner R. PROMISCUOUS: a database for network-based drugrepositioning. Nucleic Acids Res 2011; 39: D1060-6.

[116] Davis AP, King BL, Mockus S, et al. The Comparative Toxicogenomics Database: update 2011. Nucleic Acids Res 2011, 39: D1067-72.

[117] Meslamani J, Rognan D, Kellenberger E. sc-PDB: a database for identifying variations and multiplicity of 'druggable' binding sites in proteins. Bioinformatics 2011;27: 1324-6. 